\title{
The association of the circle of Willis anomaly and risk of stroke in patients with carotid artery disease
}

\author{
A associação de anomalia de Círculo de Willis e o risco de AVC em pacientes \\ com doença carotídea \\ Eylem Özaydın Göksu', Pınar Koç², Elif Küçükseymen¹, Ali Ünal³, Fatma Genç¹, Elif Sarıönder Gencer¹, Aylin Yaman¹
}

\begin{abstract}
The circle of Willis is an important collateral system that maintains perfusion to the stenotic area from the contralateral carotid and basilar artery to the region of reduced brain perfusion. The aim of the present study was to compare the circle of Willis anomaly in patients with unilateral symptomatic and asymptomatic carotid artery disease. Results: In this retrospective study, we analyzed 175 patients who presented at our outpatient stroke clinic between January, 2013 and June, 2015 with either unilateral symptomatic or asymptomatic carotid artery disease, and who had had CT angiography imaging performed. Demographic properties, carotid artery stenosis and the anomaly of the circle of Willis was recorded. Conclusion: There was no statistically significant difference in patients with symptomatic and asymptomatic carotid artery disease in terms of the anomaly of the circle of Willis.
\end{abstract}

Keywords: circle of Willis; carotid artery diseases; stroke; angiography.

RESUMO

O Círculo de Willis é um importante sistema colateral que mantém a perfusão à área estenótica da carótida contralateral e da artéria basilar para a região de perfusão cerebral reduzida. O objetivo do presente estudo foi comparar a anomalia do Círculo de Willis em pacientes com doença carotídea assintomática e sintomática unilateral. Resultados: Neste estudo retrospectivo, foram analisados 175 pacientes que foram à nossa clínica ambulatorial de AVC, entre janeiro de 2013 e junho de 2015, com doença carotídea assintomática ou sintomática unilateral, e que fizeram angiografia por tomografia computadorizada. Propriedades demográficas, estenose da artéria carótida e anomalia do Círculo de Willis foram registradas. Conclusão: Não houve diferença estatisticamente significativa em pacientes com doença carotídea sintomática e assintomática em termos de anomalia do Círculo de Willis.

Palavras-chave: círculo de Willis; doença das artérias carótidas; acidente vascular cerebral; angiografia.

Extracranial atherosclerotic disease accounts for $15-20 \%$ of all ischemic strokes ${ }^{1}$. In patients with carotid atherosclerosis, the risk of stroke is altered by the presence of collateral circulation and by the varying demographic properties of the patients ${ }^{2}$. The incidence of stroke in patients with asymptomatic and symptomatic internal carotid artery stenosis is $1,2-5,9 \%$ and $10 \%$ respectively $^{1}$.

The protective role of collateral circulation depends on many factors such as anatomic variations, systemic arterial pressure, age and the progression rate of the occlusive disease. The mainstay of collateral circulation is the circle of Willis and, in the case of a large artery occlusion, it is readily available to restore the perfusion. The anatomic properties of the collateral circulation may determine the subtypes of stroke in patients with occlusive carotid artery disease. In a previous study, up to $50 \%$ of anatomical variations in the cerebral collateral of patients were detected in the circle of Willis ${ }^{2}$.

In patients with symptomatic carotid artery stenosis, reduction of blood flow due to a stenotic carotid artery is compensated by the increased blood flow of the collateral system $^{3}$. The circle of Willis is an important collateral pathway, as it can maintain blood flow from the contralateral carotid and basilar artery to the stenotic carotid artery region. In a study of patients with symptomatic carotid artery disease,

\footnotetext{
${ }^{1}$ Antalya Education and Research Hospital, Department of Neurology, Antalya, Turkey;

${ }^{2}$ Antalya Education and Research Hospital, Department of Radiology, Antalya, Turkey;

${ }^{3}$ Akdeniz University, Department of Neurology, Antalya, Turkey.

Correspondence: Eylem Özaydın Göksu; Antalya Education and Research Hospital, Department of Neurology, Antalya, Turkey; E-mail: eylemozaydin@hotmail.com Conflict of interest: There is no conflict of interest to declare.

Received 18 July 2016; Received in final form 20 January 2017; Accepted 14 February 2017.
} 
an incomplete circle of Willis was associated with a higher risk of both transient ischemic attack and ischemic stroke ${ }^{4}$. However, the results of certain other studies indicated that the prevalence of discontinuity in the circle of Willis in patients with symptomatic carotid disease delivered conflicting results. Early autopsy studies showed that the prevalence of absent or hypoplastic segments were associated with increased stroke risk when compared with normal subjects. Conversely, other non-invasive imaging studies showed no difference in patients experiencing transient ischemic attack or ischemic stroke due to carotid disease $\mathrm{e}^{3}$.

The aim of the present study was to assess the anomalies in the circle of Willis with CT angiography in patients with symptomatic and asymptomatic carotid artery disease, to reveal its association with ischemic stroke.

\section{METHODS}

A retrospective analysis of patients who presented at our outpatient stroke clinic with carotid artery disease was conducted between January, 2013 and June, 2015. The study was approved by the ethics committee, and included 175 patients who had one-sided carotid artery disease detected by multi-detector CT angiography (Philips Healthcare, 5680 DA Best, The Netherlands). Grading of the carotid artery stenosis was performed according to the criteria of the North American Symptomatic Carotid Endarterectomy Trial ${ }^{5}$. Patients excluded from the study were those with $>50 \%$ stenosis of the contralateral carotid artery to the symptomatic carotid artery detected by CT angiography, intracranial aneurysm, vascular malformations, and patients with dissection.

The demographic properties of the patients (age, gender), the risk factors for stroke, the degree of carotid artery stenosis, whether the patients were symptomatic or asymptomatic, and the findings of the circle of Willis were recorded.

The symptomatic patients were defined as those patients who presented with the symptoms of transient ischemic attack or ischemic stroke at the vascular territory of the internal carotid artery and monocular blindness. Asymptomatic patients were defined as those patients who followed up at the outpatient stroke clinic without displaying the symptoms of large artery disease.

The circle of Willis of the patients was evaluated as the anterior communicating artery (AcomA), right and left precommunicating arteries (ACA A1 segment), posterior communicating artery (PcomA) and precommunicating posterior cerebral artery (PCA P1 segment). The hypoplastic arteries, or the arteries that could not be visualized with $\mathrm{CT}$ angiography, were accepted as abnormal.

The presence of Al segment of asymptomatic ACA, Acom A, A1 segment of symptomatic ACA, P1 segment of symptomatic PCA, symptomatic PcomA was accepted as a

complete circle of Willis, and thus the absence of any of these arteries was accepted as a circle of Willis anomaly.

\section{CT angiography}

A CT angiography examination was performed using the Philips Brilliance 64 detector CT (Holland) device (Philips Healthcare, 5680 DA Best, The Netherlands). Venous access was established through the antecubital vein and then an $80 \mathrm{~mL}$ non-ionic contrast agent was administered at a rate of $4.5 \mathrm{~mL} /$ second, while axial-plane computed tomography images of the carotid and cerebral arteries were obtained using the tracking method. Acquired slices were transferred to the workstation (Philips IntelliSpace Portal, Philips Healthcare) and multiplane images, maximum intensity projection and volume rendering 3-dimensional images were developed by postprocessing the original slices via the appropriate software (AVA). These images were reviewed with respect to the vascular plaques and stenosis.

\section{Statistical analysis}

The study data were analyzed in SPSS 16.0 for Windows (SPSS Inc., Chicago, Illinois, USA). Demographic and baseline characteristics were summarized as a mean \pm SD for continuous variables and as a percentage of the group for categorical variables. Non-normally-distributed data are presented as medians (inter-quartile range). The normality analysis was performed with the Kolmogorov-Smirnov test. Comparison of the two groups was performed by the Mann-Whitney $\mathrm{U}$ test for nonparametric comparison and the $\chi 2$ test for categorical variables. All the hypotheses were constructed as two-tailed and the $\alpha$ critical value was accepted as 0.05 .

\section{RESULTS}

During the study period, a total of 175 patients participated in this study, of whom 121 (69\%) were males and 54 (31\%) were females. The mean age of the study population was $66.7 \pm 9.2$ years. Of the 121 patients, $29.7 \%$ did not have an anomaly in the circle of Willis, while $70.3 \%$ exhibited an anomaly. Table 1 shows the demographic properties of patients with and without a circle of Willis anomaly.

Table 1. Demographic properties.

\begin{tabular}{lccc}
\hline Variable & Anomaly (+) & Anomaly (-) & p-value \\
\hline HT & $73.2 \%$ & $57.7 \%$ & 0.04 \\
DM & $39 \%$ & $28.8 \%$ & 0.20 \\
CVA & $8.9 \%$ & $9.6 \%$ & 0.88 \\
CAD & $40.7 \%$ & $34.6 \%$ & 0.45 \\
PAD & $4.9 \%$ & $1.9 \%$ & 0.36 \\
HPL & $18.7 \%$ & $34.4 \%$ & 0.02 \\
Smoker & $26 \%$ & $28.8 \%$ & 0.69 \\
\hline HT: hypertension; DM: Diabetes & mellitus; CVA: Cerebrovascular attack; \\
CAD: Coronary & Artery & Disease; PAD: Peripheral Artery & Disease; HPL: \\
Hyperlipidemia. & &
\end{tabular}


Hypertension was the most common comorbid condition in groups, followed by coronary artery disease, diabetes mellitus, smoking, hyperlipidemia, previous stroke and peripheral artery disease. The presence of hypertension $(p=0.044)$ and hyperlipidemia $(p=0.02)$ was statistically significant between the circle of Willis anomaly positive and negative patients. The numbers of patients with symptomatic and asymptomatic carotid artery disease were $83(47.4 \%)$ and $92(52.6 \%)$ respectively. An anomaly in the circle of the Willis was detected in 63 (68.7\%) patients with asymptomatic and in $60(72.3 \%)$ patients with symptomatic carotid artery disease (Tables 2 and 3). The overall incidence of anomaly in the circle of Willis was not statistically significant between the patients with symptomatic and asymptomatic carotid artery disease $(p=0.58)$. Moreover, there was no statistically significant difference in patients with symptomatic and asymptomatic carotid artery disease according to the segments of the circle of Willis (Acoma $p=0.96$, IpcomA $p=0.300$, IACA A1 $p=0.394$, IPCA P1 $p=0.256$, KACA A1 $p=0.194$, KPCA P1 $\mathrm{p}=0.448$, KPcomA $\mathrm{p}=0.473$ ).

There was a statistically significant difference between symptomatic carotid artery disease and the degree of stenosis $(\mathrm{p}<0.001)$ (Table 3). The median carotid artery stenosis in symptomatic and asymptomatic carotid artery stenosis patients was $70-90 \%$ (25th percentile 70-90\% and 75th percentile preocclusive for the symptomatic and 25th percentile 50-69\% and 75th percentile 70-90\% for the asymptomatic carotid artery stenosis group). The preocclusive and occlusive carotid artery stenosis comprised $37.4 \%$ of the symptomatic and $10.8 \%$ of the asymptomatic carotid artery disease patients.

Table 2. The association between symptomatic and asymptomatic carotid artery disease and a circle of Willis anomaly.

\begin{tabular}{lccc} 
Variable & $\begin{array}{c}\text { Anomaly negative } \\
\mathrm{n}(\%)\end{array}$ & $\begin{array}{c}\text { Anomaly positive } \\
\mathrm{n}(\%)\end{array}$ & $\mathrm{p}$-value \\
\hline Asymptomatic & $29(31.5)$ & $63(68.5)$ & 0.58 \\
Symptomatic & $23(27.7)$ & $60(72.3)$ & \\
\hline
\end{tabular}

Table 3. Circle of Willis anomaly of individual arteries between asymptomatic $(n=92)$ and symptomatic $(n=83)$ carotid artery disease.

\begin{tabular}{lcc}
\hline Variable & $\begin{array}{c}\text { Asymptomatic } \\
\mathrm{n}(\%)\end{array}$ & $\begin{array}{c}\text { Symptomatic } \\
\mathrm{n}(\%)\end{array}$ \\
\hline AcomA & $18(19.6 \%)$ & $16(19.3 \%)$ \\
IACA 11 & $19(20.7 \%)$ & $13(15.7 \%)$ \\
IPCA P1 & $7(7.6 \%)$ & $3(3.6 \%)$ \\
IPcomA & $46(50 \%)$ & $48(57.8 \%)$ \\
CACA A1 & $2(2.2 \%)$ & $5(6 \%)$ \\
CPCA P1 & $6(6.5 \%)$ & $8(9.6 \%)$ \\
CPcomA & $57(62 \%)$ & $47(56.6 \%)$ \\
\hline
\end{tabular}

AcomA: anterior communicating artery; IACA A1: ipsilateral precommunicating arteries A1 segment; CACA A1: contralateral precommunicating arteries A1 segment; IPcomA: ipsilateral posterior communicating artery; CPcomA contralateral posterior communicating artery; IPCA P1: ipsilateral precommunicating posterior cerebral artery P1 segment; CPCA P1: contralateral posterior communicating artery P1 segment.

\section{DISCUSSION}

In this study, no difference could be detected in patients with symptomatic and asymptomatic carotid artery disease in terms of the circle of Willis anomaly.

The clinical presentation of an occlusive carotid artery disease is highly variable; while some patients may be diagnosed incidentally, other patients may present with a devastating stroke. In addition, the involvement of intracranial and/or extracranial vessels may accompany this situation. The presence and effectiveness of the collateral circulation may determine the variability of clinical symptoms. The collateral cerebral circulation may not only provide sufficient blood flow to the ischemic area, but also perfuse distal to the occluded artery. In addition, the anatomical features of collateral circulation may determine the stroke type in cases of occlusive carotid artery disease. However, it is reported that up to $50 \%$ of significant anatomic variations among patients were seen in the circle of Willis ${ }^{2}$.

The contribution of the circle of Willis to cerebral hemodynamics is not only determined by the stenosis of vertebral and carotid arteries, but also by the presence of the vessels that constitute the circle of Willis.

Previous studies indicated that patients with asymptomatic internal carotid artery occlusions have a better preserved hemodynamic status when compared with symptomatic patients ${ }^{6,7}$. However, from this perspective, the role of the circle of Willis is not clear.

In the study conducted by Waajer et al., instances of the circle of Willis anomaly were significantly higher in patients with symptomatic carotid disease when compared to the control patients. In their study, they found significantly more hypoplastic or invisible A1 segments in the symptomatic carotid artery stenosis group, and the compromised anterior collateral pathway and usually-accompanying posterior pathway occurred more frequently in the symptomatic carotid artery stenosis group. In this study, controls were retrospectively collected from patients without carotid stenosis. As we had no control group in our study, we could not draw this conclusion ${ }^{3}$. In an magnetic resonance angiography study by Hartkamp et al., a significantly higher percentage of complete anterior and posterior circulation configuration was found in the patient group than in the control subjects ${ }^{8}$. The prevalence of a circle of the Willis anomaly was reported in $79.7 \%$ of the symptomatic carotid artery disease group 9 . In our study, the anomaly in the circle of the Willis was $72.3 \%$ and $68.5 \%$ in the symptomatic and asymptomatic carotid artery disease groups respectively. Although we did not recruit a healthy control group for our study, the prevalence we have reported in this study is significantly higher than the prevalence of $50 \%$ in the normal population ${ }^{9,10}$. Although the number of patients with a circle of the Willis anomaly is more significant in the symptomatic carotid artery disease group, there was no statistically 
significant difference among the symptomatic and asymptomatic carotid artery disease groups.

In a prior study, it was thought that the hypoplasia of the ACA Al segment may be a factor that contributes to acute ischemic stroke, due to compromise of the collateral circulation. When compared with healthy individuals, the anomaly was detected significantly more often in acute ischemic stroke patients ${ }^{11}$. In a study conducted by Shaban et al., hypoplastic and absent ACA A1 were detected in 5.9\% of acute ischemic stroke patients, although hypoplastic and absent ACA A1 did not have any effect on vascular distribution, side, or the volume of the infarct ${ }^{10}$. In our study, an ipsilateral or contralateral presence of the ACA Al segment was not significantly different between the symptomatic and asymptomatic patient population. (IACA A1 $\mathrm{p}=0.394$, KACA A1 p = 0.194).

In patients with unilateral internal carotid artery occlusion, the presence of collateral flow via the posterior communicating artery in the circle of the Willis was associated with a low prevalence of border zone infarcts ${ }^{12}$. However, collateral function of the circle of the Willis was not increased in asymptomatic patients with internal carotid artery occlusion. In our study, absence of ipsilateral PcomA was not statistically significant different between the symptomatic and asymptomatic groups $(\mathrm{p}=0.300)$.

The cerebral collateral circulation maintains cerebral tissue perfusion under physiologic and pathologic conditions, such as ischemia. Previous studies revealed the role of collateral circulation in patients with carotid artery disease: the absence of collateral circulation in patients with symptomatic carotid artery disease has a worse prognosis. Evaluation of the cerebral collateral circulation, together with the cerebral perfusion and the clinical parameters of patients with occlusive carotid artery disease, may give the full spectrum of the cerebrovascular condition. Nevertheless, the clinical applications of information regarding collateral circulation in patients with carotid artery disease are still not clear ${ }^{2}$.

Our study has several limitations, such as its retrospective design, the limited number of patients and the lack of a control group.

In conclusion, we were able to detect a difference between the symptomatic and asymptomatic internal carotid artery occlusion patient population in terms of a circle of the Willis anomaly. Future prospective studies may, therefore, help to better understand the role of collateral circulation in patients with carotid artery disease.

\section{References}

1. Gupta A, Chazen JL, Hartman M, Delgado D, Anumula N, Shao $\mathrm{H}$ et al. Cerebrovascular reserve and stroke risk in patients with carotid stenosis or occlusion: a systematic review and meta-analysis. Stroke. 2012;43(11):2884-91. https://doi.org/10.1161/STROKEAHA.112.663716

2. Romero JR, Pikula A, Nguyen TN, Nien YL, Norbash A, Babikian VL. Cerebral collateral circulation in carotid artery disease. Curr Cardiol Rev. 2009;5(4):279-88. https://doi.org/10.2174/157340309789317887

3. Waaijer A, Leeuwen MS, Worp HB, Verhagen HJ, Mali WP, Velthuis BK. Anatomic variations in the circle of Willis in patients with symptomatic carotid artery stenosis assessed with multidetector row CT angiography. Cerebrovasc Dis. 2007;23(4):267-74. https://doi.org/10.1159/000098326

4. Henderson RD, Eliasziw M, Fox AJ, Rothwell PM, Barnett HJ. Angiographically defined collateral circulation and risk of stroke in patients with severe carotid artery stenosis. Stroke. 2000;31(1):128-32. https://doi.org/10.1161/01.STR.31.1.128

5. North American Symptomatic Carotid Endarterectomy Trial (NASCET) investigators. Clinical alert: benefit of carotid endarterectomy for patients with high-grade stenosis of the internal carotid artery. Stroke. 1991;22(6):816-7. https://doi.org/10.1161/01.STR.22.6.816

6. Hoksbergen AW, Legemate DA, Csiba L, Csáti G, Síró P, Fülesdi B et al. Absent collateral function of the circle of Willis as risk factor for ischemic stroke. Cerebrovasc Dis. 2003;16(3):191-8. https://doi.org/10.1159/000071115

7. Bozzao A, Floris R, Gaudiello F, Finocchi V, Fantozzi LM, Simonetti G. Hemodynamic modifications in patients with symptomatic unilateral stenosis of the internal carotid artery: evaluation with MR imaging perfusion sequences. AJNR Am J Neuroradiol. 2002;23(8):1342-5.

8. Hartkamp MJ, Der Grond JV, Everdingen KJ, Hillen B, Mali WP. Circle of Willis collateral flow investigated by magnetic resonance angiography .Stroke. 1999;30(12)2671-8. https://doi.org/10.1161/01.STR.30.12.2671

9. Kamıșlı S, Kamıșı Ö, Teker U, Kablan Y, Saraç K, Özcan C. [Circle of Willis anomalies in stroke patients related with symptomatic carotid artery disease]. Turkish J Cerebrovasc Dis. 2012;18(1):6-9. Turkish https://doi.org/10.5505/tbdhd.2012.36844

10. Shaban A, Albright K, Gouse B, George A, Monlezun D, Boehme $A$ et al. The impact of absent $A 1$ segment on ischemic stroke characteristics and outcomes. J Stroke Cerebrovasc Dis. 2015;24(1):171-5. https://doi.org/10.5505/tbdhd.2012.36844

11. Chuang YM, Liu CY, Pan PJ, Lin CP. Anterior cerebral artery A1 segment hypoplasia may contribute to A1 hypoplasia syndrome. Eur Neurol. 2007;57(4):208-11. https://doi.org/10.1159/000099160

12. Hendrikse J, Hartkamp MJ, Hillen B, Mali WP, Grond J. Collateral ability of the circle of Willis in patients with unilateral internal carotid artery occlusion: border zone infarcts and clinical symptoms. Stroke. 2001;32(12):2768-73. https://doi.org/10.1161/hs1201.099892 